\title{
High-Selenium Baker's Yeast
}

National Cancer Institute

\section{Source}

National Cancer Institute. High-Selenium Baker's Yeast. NCI Thesaurus. Code C88269.

A baker's yeast (Saccharomyces cerevisiae) containing high levels of the trace element selenium (Se) with potential chemopreventive, immunomodulating and antioxidant activities. Selenium is introduced into yeast during fermentation and incorporated into selenocompounds, such as selenomethionine and glutamyl SE methylselenocysteine. Selenium functions as a cofactor for antioxidant enzymes such as glutathione peroxidases and thioredoxin reductase. 